\title{
Set-Membership Filtering for Time-Varying Systems with Mixed Time-Delays under Round-Robin and Weighted Try-Once-Discard Protocols
}

\author{
Lei Zou ${ }^{a}$, Zidong Wang a,b, Huijun Gao ${ }^{c}$ \\ ${ }^{a}$ College of Electrical Engineering and Automation, Shandong University of Science and Technology, Qingdao 266590, China. \\ ${ }^{\mathrm{b}}$ Department of Computer Science, Brunel University London, Uxbridge, Middlesex, UB8 3PH, United Kingdom. \\ ${ }^{\mathrm{c}}$ Research Institute of Intelligent Control and Systems, Harbin Institute of Technology, Harbin 150001, China.
}

\begin{abstract}
This paper is concerned with the set-membership filtering problem for a class of time-varying systems with mixed time-delays and communication protocols. Two kinds of well-known protocols (Round-Robin protocol and Weighted Try-Once-Discard protocol) are considered, with which the data transmission between the sensor nodes and the filter is implemented via a shared communication network that allows only one sensor node to send its measurement data at each transmission instant in order to prevent the data from collisions. The transmission order of sensor nodes is orchestrated by the underlying protocol of the network. The aim of the problem addressed is to design a set-membership filter capable of confining the state estimate of the system to certain ellipsoidal region subject to the bounded non-Gaussian noises. Sufficient condition is first derived for the existence of the desired filter at each time step in terms of a recursive algorithm. Then, two optimization problems are solved by optimizing the constraint ellipsoid of the estimation error subject to the underlying protocol. Simulation results demonstrate the effectiveness of the proposed filter design scheme.
\end{abstract}

Key words: Communication protocol; Set-membership filtering; Round-Robin protocol; Weighted Try-Once-Discard protocol; Mixed time-delays; Non-Gaussian noises; Recursive matrix inequalities.

\section{Introduction}

The past decades have witnessed a surge of research interest on networked systems due primarily to their extensive applications in various fields including environmental monitoring, industrial automation, smart grids and distributed/mobile communications. The key feature of networked systems is that the connections of system components are implemented via shared communication networks. Networked systems possess many advantages such as low cost, simple installation, reduced system wiring and high reliability. Compared with the traditional systems with point-to-point communication scheme, the utilization of communication network has led to rich yet complex network-induced behaviors (e.g. communication delays, packet dropouts and signal quantization) and these behaviors have attracted considerable research attention, see e.g. [5, 13, 20, 21, 27].

Filtering problem is a fundamental research issue in signal processing and control communities $[1,4,10,12,23$,

* This work was supported in part by the Research Fund for the Taishan Scholar Project of Shandong Province of China, the National Natural Science Foundation of China under Grants 61329301, 61273156 and 61333012, the Royal Society of the U.K., and the Alexander von Humboldt Foundation of Germany. Corresponding author Z. Wang.

Email address: Zidong.Wang@brunel.ac.uk (Zidong Wang).
26,28]. For linear (or nonlinear) systems with Gaussian noises, the Kalman filtering (or extended Kalman filter) method is always recognized as a reliable filtering approach. Nevertheless, the Kalman filter may lead to unsatisfactory performance when the disturbances are nonGaussian noises. Another well-known filtering method is the $\mathcal{H}_{\infty}$ filtering approach whose aim is to guarantee a given disturbance attenuation level on the estimation error subject to energy-bounded noises. For conventional $\mathcal{H}_{\infty}$ filtering method, there does not seem to be any provision to ensure the boundedness of the estimation error. In this regard, the so-called set-membership filtering method does well as it generates a satisfactory state estimate guaranteeing that the estimation error is confined to a bounded region in the state-space subject to the unknown-but-bounded noises. The origination of the set-membership filtering problem dated back to the 1960s and such a problem has gained recurring research interest in the past decade $[9,19,24,25]$.

In most existing literature concerning the filtering problems of networked systems, it has been assumed that all the sensor nodes could simultaneously get access to the network to transmit signals. This assumption, however, is generally unrealistic for networked systems since realworld networks unavoidably suffer from limited bandwidth which is likely to give rise to data collisions in case of simultaneous multiple accesses. As such, the communication protocols are needed to orchestrate the trans- 
mission order of sensor nodes $[2,22,29]$. The widely utilized protocols in industry include, but are not limited to, the Round-Robin (RR) protocol [17], the Weight Try-Once-Discard (WTOD) protocol [7] and stochastic communication protocol $[14,16]$. Compared with those traditional schemes without protocol scheduling, the utilization of communication protocol would bring in certain fundamental challenges (or protocol-induced effects) to the dynamics analysis issues. As such, it is necessary to examine how the inclusion of the communication protocol impacts on the control and filtering problems of networked systems. So far, some preliminary result$\mathrm{s}$ have been reported on the analysis issue of networked systems subject to communication protocols.

Among various communication protocols, the RR and WTOD protocols are widely employed by communication and signal processing communities. The RR protocol is also known as the time-division multiple access (TDMA) protocol or Token Ring protocol. Under the scheduling of the RR protocol, the transmission instants of all the sensor nodes are predetermined according to a fixed circular order. Obviously, the RR protocol is a periodic protocol. On the other hand, the WTOD protocol belongs to the class of quadratic protocols. Different from the "periodic assignment" behavior of the RR protocol, the WTOD protocol assigns the transmission instants to certain sensor nodes according to a given quadratic selection principle. For the purpose of characterizing the scheduling behaviors caused by protocol$\mathrm{s}$, the switched-linear-system framework has been introduced in [7] for discrete-time networked systems in which both the RR and the WTOD scheduling behaviors have been considered. Based on the switched-linear-system framework, the co-design problem of both the stabilizing controller and scheduling protocol has been investigated in [15] for a class of networked control systems with multiple distributed transmission delays.

On the other hand, it is well known that time-delays exist widely in practice and may cause undesirable dynamic behaviors including oscillation and instability. In recent years, a rich body of literature has appeared on the control and filtering problems of networked systems with time-delays, see e.g. [11] and the references therein. Unfortunately, when it comes to the set-membership filtering problems with time-delays under communication protocols, the corresponding results have been really scattered due mainly to the difficulties in handling the coupling issues between the set membership and the scheduling protocols. To this end, a seemingly interesting research problem that is of clear engineering insight is to investigate the set-membership filtering problem for the time-varying networked systems with a communication protocol (the RR protocol or WTOD protocol), and this has motivated our present research.

Summarizing the above discussions, in this paper, we aim to deal with the set-membership filtering problem for the time-varying networked system with simultaneous presence of mixed time-delays and communication protocol scheduling. More specifically, the objective of this paper is to design a set-membership filter for the networked systems with mixed time-delays subject to the RR protocol and WTOD protocol, respectively. The main contributions of this paper are highlighted as follows: 1) the set-membership filtering problem is, for the first time, investigated for time-varying systems with the protocol scheduling; 2) the influences from both the $R$ $R$ protocol and the WTOD protocol on the filter performance are considered; and 3) the filter gain matrix is obtained by solving a set of recursive matrix inequalities that are solvable via standard software package.

Notations: The notation used here is fairly standard except where otherwise stated. $\mathbb{R}^{n}$ and $\mathbb{R}^{n \times m}$ denote, respectively, the $n$ dimensional Euclidean space and set of all $n \times m$ real matrices. $\mathbb{N}\left(\mathbb{N}^{+}, \mathbb{N}^{-}\right)$denotes the set of integers (nonnegative integers, negative integers). The notation $X \geq Y(X>Y)$, where $X$ and $Y$ are real symmetric matrices, means that $X-Y$ is positive semi-definite (positive definite). Prob $\{\cdot\}$ means the occurrence probability of the event ".". $\mathbb{E}\{x\}$ and $\mathbb{E}\{x \mid y\}$ will, respectively, denote the expectation of the stochastic variable $x$ and expectation of $x$ conditional on $y$. 0 represents the zero matrix of compatible dimensions. The $n$-dimensional identity matrix is denoted as $I_{n}$ or simply $I$, if no confusion is caused. The shorthand $\operatorname{diag}\{\ldots\}$ stands for a block-diagonal matrix. $\|A\|$ refers to the norm of a matrix $A$ defined by $\|A\|=\sqrt{\operatorname{trace}\left(A^{T} A\right)} \cdot M^{T}$ represents the transpose of $M$. In symmetric block matrices, "*" is used as an ellipsis for terms induced by symmetry. The Kronecker delta function $\delta(a)$ is a binary function that equals 1 if $a=0$ and equals 0 otherwise. Matrices, if they are not explicitly specified, are assumed to have compatible dimensions.

\section{Problem Formulation and Preliminaries}

In this section, we first introduce some preliminaries related to the Round-Robin (RR) protocol and the Weighted Try-Once-Discard (WTOD) protocol, and then describe the problem setup.

\subsection{Communication Protocols}

Consider a networked system with $N$ nodes labeled as $\{1,2, \cdots, N\}$. In this system, all the nodes transmit their data via a shared communication network in which only one node is allowed to get access to the network at each transmission instant for the purpose of preventing the data from collisions. Let $\xi(k) \in\{1,2, \cdots, N\}$ be the selected node obtaining access to the communication network at time instant $k$. The value of $\xi(k)$ is determined by the communication protocol of the network.

\section{1) The Round-Robin (RR) Protocol:}

Under the scheduling of the RR protocol, the value of $\xi(k)$ satisfies $\xi(k+N)=\xi(k)$ for all $k \in \mathbb{N}^{+}$and $\xi(k)=$ $k$ for $k \in\{1,2, \cdots, N\}$. In other words, $\xi(k)$ can be calculated as:

$$
\xi(k)=\bmod (k-1, N)+1 .
$$


In such a protocol, the number of nodes $N$ can be regarded as the period of the RR protocol. During each period of the RR protocol, each node has access to the communication network exactly once.

2) The Weighted Try-Once-Discard (WTOD) Protocol:

WTOD protocol is a dynamical protocol in which the value of $\xi(k)$ is determined by the following selection principle:

$$
\xi(k) \triangleq \arg \max _{1 \leq i \leq N}\left(y_{i}(k)-y_{i}^{*}(k)\right)^{T} Q_{i}\left(y_{i}(k)-y_{i}^{*}(k)\right)
$$

where $y_{i}^{*}(k)$ represents the last transmitted signal before time instant $k$ of node $i$ and $Q_{i}(i \in\{1,2, \cdots, N\})$ is a known positive definite matrix denoting the weight matrix of the $i$-th node.

Define $y(k) \triangleq\left[y_{1}^{T}(k) y_{2}^{T}(k) \cdots y_{N}^{T}(k)\right]^{T}$ and $y^{*}(k) \triangleq$ $\left[\left(y_{1}^{*}(k)\right)^{T}\left(y_{2}^{*}(k)\right)^{T} \cdots\left(y_{N}^{*}(k)\right)^{T}\right]^{T}$. The selection principle (2) could be rewritten as

$$
\xi(k)=\arg \max _{1 \leq i \leq N}\left(y(k)-y^{*}(k)\right)^{T} \bar{Q}_{i}\left(y(k)-y^{*}(k)\right)
$$

where $\bar{Q}=\operatorname{diag}\left\{Q_{1}, Q_{2}, \cdots, Q_{N}\right\}, \bar{Q}_{i}=\bar{Q} \Phi_{i}, \Phi_{i}=$ $\operatorname{diag}\{\delta(i-1) I, \delta(i-2) I, \cdots, \delta(i-N) I\}(1 \leq i \leq N)$ and $\delta(\cdot) \in\{0,1\}$ is the Kronecker delta function.

\subsection{Problem Formulation}

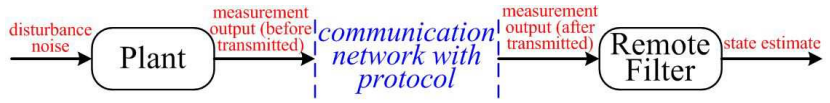

Fig. 1. Filtering problem for a networked system subject to a communication protocol

Consider a filtering problem for a networked system shown in Fig. 1. In this framework, the signal transmission between the remote filter and the sensors of the plant is implemented through a communication network. In the following, let us introduce the plant and the communication network in a mathematical way. The plan$\mathrm{t}$ under consideration is a discrete time-varying system with mixed time-delays of the form:

$$
\left\{\begin{aligned}
x(k+1)= & A(k) x(k)+E(k) x\left(k-\tau_{1}\right) \\
& +F(k) \sum_{i=1}^{\tau_{2}} \mu_{i} x(k-i)+B(k) \omega(k) \\
y(k)= & C(k) x(k)+D(k) \nu(k) \\
x(i)= & \psi_{1}(i), \quad-\max \left\{\tau_{1}, \tau_{2}\right\} \leq i \leq 0
\end{aligned}\right.
$$

where $x(k) \in \mathbb{R}^{n_{x}}$ and $y(k) \in \mathbb{R}^{n_{y}}$ denote, respectively, the state vector and the measurement output before transmitted through the communication network. The parameters $A(k), E(k), F(k), B(k), C(k)$ and $D(k)$ are real-valued time-varying matrices of appropriate dimensions. $\mu_{i}\left(1 \leq i \leq \tau_{2}\right), \tau_{1}$ and $\tau_{2}$ are known positive integers. $\omega(\bar{k}) \in \mathbb{R}^{n_{\omega}}$ and $\nu(k) \in \mathbb{R}^{n_{\nu}}$ represent the process and measurement noises, respectively. $\psi_{1}(i)$ $\left(i=-\max \left\{\tau_{1}, \tau_{2}\right\},-\max \left\{\tau_{1}, \tau_{2}\right\}+1, \cdots, 0\right)$ are the initial conditions.

Assumption 1 The noise sequences $\omega(k)$ and $\nu(k)$ are confined to the following ellipsoidal sets:

$$
\left\{\begin{array}{c}
\mathscr{W}_{k} \triangleq\left\{\omega(k): \omega^{T}(k) S^{-1}(k) \omega(k) \leq 1\right\} \\
\mathscr{V}_{k} \triangleq\left\{\nu(k): \nu^{T}(k) R^{-1}(k) \nu(k) \leq 1\right\}
\end{array}\right.
$$

where $S(k)$ and $R(k)$ are known positive definite matrices with compatible dimensions.

Remark 1 As is well known, in practical engineering, disturbance noises are known to be non-Gaussian due to man-made electromagnetic interference and other natural sources, see, e.g. [8]. As such, most conventional filtering approaches, such as the well-known Kalman filtering, are no longer applicable to the filtering problems subjec$t$ to non-Gaussian noises. In this paper, the process and measurement noises are assumed to be unknown, bounded, deterministic but reside within certain ellipsoidal sets. Matrices $S(k)$ and $R(k)$ can be regarded as "special" upper-bounds on the amplitude of the noises.

We are now ready to introduce the signal transmission of the measurement output. Without loss of generality, it is assumed that sensors of the system are grouped into $N$ sensor nodes according to their spatial distribution. As such, for technical analysis, the measurement output before transmitted can be rewritten as

$$
y(k)=\left[\begin{array}{lllll}
y_{1}^{T}(k) & y_{2}^{T}(k) & \cdots & y_{N}^{T}(k)
\end{array}\right]^{T}
$$

where $y_{i}(k)(i \in\{1,2, \cdots, N\})$ is the measurement of the $i$-th sensor node before transmitted.

The communication between the sensors and the remote filter is scheduled by certain communication protocol. In this paper, we aim to study the set-membership filtering problem subject to two different kinds of communication protocols, namely, the RR protocol and the WTOD protocol. For the sake of examining the influence of communication protocols, let $\xi(k) \in\{1,2, \cdots, N\}$ be the selected sensor node obtaining access to the communication network at time instant $k$. As shown in subsection $2.1, \xi(k) \in\{1,2, \cdots, N\}$ is a switching function dependent on the underlying protocol scheduling. The value of $\xi(k)$ is determined according to (1) in the case of RR scheduling and (3) in the case of WTOD scheduling.

Next, let us denote the measurement output after transmitted through the network by

$$
\bar{y}(k) \triangleq\left[\bar{y}_{1}^{T}(k) \bar{y}_{2}^{T}(k) \cdots \bar{y}_{N}^{T}(k)\right]^{T} \in \mathbb{R}^{n_{y}} .
$$

The initial state of $\bar{y}(k)$ is assumed to be $\bar{y}(j)=\psi_{2}$ for $j<0$ where $\psi_{2}$ is a known vector. The updating rule of $\bar{y}_{i}(k)\left(k \in \mathbb{N}^{+}, i \in\{1,2, \cdots, N\}\right)$ is set to be

$$
\bar{y}_{i}(k)= \begin{cases}y_{i}(k), & \text { if } i=\xi(k) \\ \bar{y}_{i}(k-1), & \text { otherwise. }\end{cases}
$$

According to the updating rule (6), it can be seen that

$$
\left\{\begin{array}{l}
\bar{y}(k)=\Phi_{\xi(k)} y(k)+\left(I_{n_{y}}-\Phi_{\xi(k)}\right) \bar{y}(k-1) \\
\bar{y}(j)=\psi_{2}, \quad j<0 .
\end{array}\right.
$$

Denoting $\bar{x}(k) \triangleq\left[x^{T}(k) \bar{y}^{T}(k-1)\right]^{T}$ and $\bar{\omega}(k) \triangleq$ $\left[\omega^{T}(k) \nu^{T}(k)\right]^{T}$, the time-varying system (4) with a protocol scheduling can be reformulated as follows: 


$$
\left\{\begin{aligned}
\bar{x}(k+1)= & \mathcal{A}(k) \bar{x}(k)+\mathcal{E}(k) \bar{x}\left(k-\tau_{1}\right) \\
& +\mathcal{F}(k) \sum_{i=1}^{\tau_{2}} \mu_{i} \bar{x}(k-i)+\mathcal{B}(k) \bar{\omega}(k) \\
\bar{y}(k)= & \mathcal{C}(k) \bar{x}(k)+\mathcal{D}(k) \bar{\omega}(k) \\
\tilde{x}(i)= & \bar{\psi}(i), \quad-\max \left\{\tau_{1}, \tau_{2}\right\} \leq i \leq 0
\end{aligned}\right.
$$

where $\bar{\psi}(i)=\left[\psi_{1}^{T}(i) \psi_{2}^{T}(i)\right]^{T}$ and

$$
\begin{aligned}
& \mathcal{A}(k)=\left[\begin{array}{cc}
A(k) & 0 \\
\Phi_{\xi(k)} C(k) & I-\Phi_{\xi(k)}
\end{array}\right], \quad \mathcal{F}(k)=\left[\begin{array}{cc}
F(k) & 0 \\
0 & 0
\end{array}\right], \\
& \mathcal{E}(k)=\left[\begin{array}{cc}
E(k) & 0 \\
0 & 0
\end{array}\right], \quad \mathcal{B}(k)=\left[\begin{array}{cc}
B(k) & 0 \\
0 & \Phi_{\xi(k)} D(k)
\end{array}\right], \\
& \mathcal{C}(k)=\left[\begin{array}{lll}
\Phi_{\xi(k)} C(k) & I-\Phi_{\xi(k)}
\end{array}\right], \mathcal{D}(k)=\left[\begin{array}{ll}
0 & \Phi_{\xi(k)} D(k)
\end{array}\right] .
\end{aligned}
$$

\subsection{Time-varying Filter}

In this paper, a filter based on the signal $\bar{y}(k)$ is considered for the augmented system (8) with mixed timedelays, which is of the form:

$$
\left\{\begin{aligned}
& \hat{x}(k+1)= \mathcal{A}(k) \hat{x}(k)+\mathcal{E}(k) x\left(k-\tau_{1}\right)+K(k) \bar{y}(k) \\
&+\mathcal{F}(k) \sum_{i=1}^{\tau_{2}} \mu_{i} \hat{x}(k-i)-K(k) \mathcal{C}(k) \hat{x}(k) \\
& \hat{x}(i)=0, \quad-\max \left\{\tau_{1}, \tau_{2}\right\} \leq i \leq 0
\end{aligned}\right.
$$

where $\hat{x}(k) \in \mathbb{R}^{n_{x}+n_{y}}$ is the state estimate of $\bar{x}(k)$ and $K(k) \in \mathbb{R}^{\left(n_{x}+n_{y}\right) \times n_{y}}$ is the filter gain to be designed.

Letting the estimation error be $e(k) \triangleq \bar{x}(k)-\hat{x}(k)$, the estimation error dynamics for the augmented system (8) can be obtained as follows:

$$
\begin{aligned}
& e(k+1)=(\mathcal{A}(k)-K(k) \mathcal{C}(k)) e(k)+\mathcal{E}(k) e\left(k-\tau_{1}\right) \\
& +\mathcal{F}(k) \sum_{i=1}^{\tau_{2}} \mu_{i} e(k-i)+(\mathcal{B}(k)-K(k) \mathcal{D}(k)) \bar{\omega}(k) .
\end{aligned}
$$

Before proceeding further, we introduce the following definition and assumption.

Definition 1 For the time-varying system (8) with filter (9), let the sequence of the constraint matrices (ellipsoid matrices) $P(k) \in \mathbb{R}^{\left(n_{x}+n_{y}\right) \times\left(n_{x}+n_{y}\right)}\left(k \in \mathbb{N}^{+}\right)$be given. The filtering error $e(k)$ is said to satisfy the $P(k)$ dependent constraint if the following set of inequalities

$$
e^{T}(k) P^{-1}(k) e(k) \leq 1
$$

hold for $k \in \mathbb{N}^{+}$.

Assumption 2 The initial conditions $\bar{\psi}(i) \quad(0 \geq i \geq$ $\left.-\max \left\{\tau_{1}, \tau_{2}\right\}\right)$ satisfy

$$
\bar{\psi}^{T}(i) P^{-1}(i) \bar{\psi}(i) \leq 1
$$

where $P(i)\left(-\max \left\{\tau_{1}, \tau_{2}\right\} \leq i \leq 0\right)$ are a known positive definite matrices.
We are now in the position to state the problem addressed in this paper as follows. The objective of this paper is to design appropriate filter parameter $K(k)$ subject to different protocols (the RR protocol and the WTOD protocol). To be more specific, we are interested in dealing with the following analysis and design problems:

Problem 1: For a given sequence of constraint matrices $P(k)$, establish sufficient conditions for the existence of the filter gains under which the dynamics of the filtering error $e(k)$ satisfies the $P(k)$-dependent constraint (11) subject to the process and measurement noises;

Problem 2: Minimize the trace of the matrix $P(k)$ by appropriately choosing the filter parameter $K(k)$ satisfying the conditions established in Problem 1.

\section{Main results}

In this section, we shall present sufficient conditions guaranteeing that the dynamics of the filtering error $e(k)$ satisfies the $P(k)$-dependent constraint (11). Furthermore, two recursive algorithms are proposed to obtain the filter parameter $K(k)$ subject to the RR protocol and the WTOD protocol, respectively. Before proceeding further, we introduce the following lemma which will be needed for the derivation of our main results.

Lemma 1 (S-procedure [3]) Let $\phi_{0}(\cdot), \phi_{1}(\cdot), \cdots, \phi_{p}(\cdot)$ be quadratic functions of the variable $\varsigma \in \mathbb{R}^{n}: \phi_{i}(\varsigma) \triangleq$ $\varsigma^{T} T_{i} \varsigma(i=0,1, \cdots, p)$ with $T_{i}^{T}=T_{i}$. If there exist $\alpha_{1}$, $\alpha_{2}, \cdots, \alpha_{p} \geq 0$ such that

then the following is true

$$
T_{0}-\sum_{i=1}^{p} \alpha_{i} T_{i} \leq 0,
$$

$$
\phi_{1}(\varsigma) \leq 0, \phi_{2}(\varsigma) \leq 0, \cdots, \phi_{p}(\varsigma) \leq 0 \Rightarrow \phi_{0}(\varsigma) \leq 0 .
$$

\section{$3.1 P(k)$-Dependent Constraint Analysis}

In the following theorems, sufficient conditions are established to solve Problem 1 that aims to ensure the $P(k)$ dependent constraint to be satisfied for the dynamics of the filtering error subject to the WTOD protocol and the RR protocol. A recursive matrix inequality approach is developed to cater for the effects from the ellipsoidal constraints, mixed time-delays and protocols.

\section{1) The WTOD Protocol}

Theorem 1 Consider the system (4), the WTOD protocol given by (3) and the filter (9). Let the sequence of constraint matrices $P(k)>0\left(k \in \mathbb{N}^{+}\right)$be given. Assume that there exist real valued matrices $K(k)$, positive scalars $\lambda_{i}(k)$ and $\varepsilon_{j}(k)\left(k \in \mathbb{N}^{+}, 1 \leq i \leq N, 1 \leq j \leq 4\right)$ satisfying the following recursive matrix inequality

where

$$
\left[\begin{array}{cc}
-\Theta(k) & \Pi^{T}(k) \\
* & -P(k+1)
\end{array}\right] \leq 0
$$


$\overline{\mathcal{A}}_{k}=\mathcal{A}(k)-K(k) \mathcal{C}(k), \quad \overline{\mathcal{B}}_{k}=\mathcal{B}(k)-K(k) \mathcal{D}(k)$,

$\overline{\mathcal{F}}_{\tau}(k)=\left[\begin{array}{llll}\mathscr{F}_{1} & \mathscr{F}_{2} & \cdots & \mathscr{F}_{\tau_{2}}\end{array}\right], \quad \mathscr{F}_{i}=\mu_{i} \mathcal{F}(k) L(k-i)$,

$\Omega(k)=\operatorname{diag}\{S(k), R(k)\}, \vec{C}(k)=[C(k)-I]$,

$\Gamma(k)=[\vec{C}(k) \hat{x}(k) \vec{C}(k) L(k) 000 \vec{D}(k)], \vec{D}(k)=\left[\begin{array}{lll}0 & D(k)\end{array}\right]$,

$\bar{\varepsilon}(k)=1-\varepsilon_{1}(k)-\varepsilon_{2}(k)-\tau_{2} \varepsilon_{3}(k)-2 \varepsilon_{4}(k)$

and $L(k)$ is the factorization of $P(k)=L(k) L^{T}(k)$.

Then, the dynamics of (10) satisfies the $P(k)$-dependent constraint with the estimator parameters $K(k)\left(k \in \mathbb{N}^{+}\right)$.

Proof: The proof of this theorem is performed by mathematical induction. In what follows, let us first prove the following assertion is true.

Assertion: The solution $e(k)$ of the time-varying system (10) satisfies

$$
e^{T}(k) P^{-1}(k) e(k) \leq 1, \quad k \in \mathbb{N}^{+} .
$$

The proof of the above assertion is divided into two steps, namely, the initial step and the inductive step.

Initial step. For $t=0$, it can be immediately known from Assumption 2 that

$$
e^{T}(0) P^{-1}(0) e(0)=\psi^{T}(0) P^{-1}(0) \psi(0) \leq 1 .
$$

Inductive step. Now that the assertion is true for $t=0$. Next, given that the assertion is true for $t \leq k$, we aim to show that the same assertion is true for $t=k+1$.

Since the assertion is true for $t \leq k$, if follows from [6] that there exists a vector $z(i)$ satisfying $e(i)=L(i) z(i)$ with $\|z(i)\| \leq 1$ where $L(i)$ is a factorization of $P(i)=$ $L(i) L^{T}(i)$ for $i \in\{0,1, \cdots, k\}$. It remains to show that, for $t=k+1$, the solution $P(k+1)$ of the inequalities (15) guarantees $e^{T}(k+1) P^{-1}(k+1) e(k+1) \leq 1$.

For notational simplicity, we first denote $\bar{z}_{\tau}(k) \triangleq$ $\left[\begin{array}{llll}z^{T}(k-1) & z^{T}(k-2) & \cdots & z^{T}\left(k-\tau_{2}\right)\end{array}\right]^{T}$ and $\eta(k) \triangleq$ $\left[1 z^{T}(k) z^{T}\left(k-\tau_{1}\right) \bar{z}_{\tau}^{T}(k) \bar{\omega}^{T}(k)\right]^{T}$. Then, we have

$$
e(k+1)=\Pi(k) \eta(k)
$$

and therefore

$$
\begin{aligned}
& e^{T}(k+1) P^{-1}(k+1) e(k+1)-1 \\
= & \eta^{T}(k)\left(\Pi^{T}(k) P^{-1}(k+1) \Pi(k)\right. \\
& -\operatorname{diag}\{1,0,0,0,0\}) \eta(k) .
\end{aligned}
$$

On the other hand, it can be obtained from Assumption 1 and the selection principle of the WTOD that

$$
\left\{\begin{array}{l}
\|z(k)\|^{2} \leq 1,\left\|z\left(k-\tau_{1}\right)\right\|^{2} \leq 1,\left\|\bar{z}_{\tau}(k)\right\|^{2} \leq \tau_{2}, \\
\bar{\omega}^{T}(k) \operatorname{diag}\left\{S^{-1}(k), R^{-1}(k)\right\} \bar{\omega}(k) \leq 2 \\
(y(k)-\bar{y}(k-1))^{T} \bar{Q}\left(\Phi_{i}-\Phi_{\xi(k)}\right) \\
\quad \times(y(k)-\bar{y}(k-1)) \leq 0, i=1,2, \cdots, N
\end{array}\right.
$$

which can be rearranged by means of $\eta(k)$ as follows:

$$
\left\{\begin{array}{l}
\eta^{T}(k) \operatorname{diag}\{-1, I, 0,0,0\} \eta(k) \leq 0, \\
\eta^{T}(k) \operatorname{diag}\{-1,0, I, 0,0\} \eta(k) \leq 0, \\
\eta^{T}(k) \operatorname{diag}\left\{-\tau_{2}, 0,0, I, 0\right\} \eta(k) \leq 0, \\
\eta^{T}(k) \operatorname{diag}\left\{-2,0,0,0, \Omega^{-1}(k)\right\} \eta(k) \leq 0, \\
\eta^{T}(k) \Gamma^{T}(k) \bar{Q}\left(\Phi_{i}-\Phi_{\xi(k)}\right) \Gamma(k) \eta(k) \leq 0
\end{array}\right.
$$

On the other hand, it can be seen from the S-procedure (Lemma 1) that if there exist positive scalars $\varepsilon_{1}(k)$, $\varepsilon_{2}(k), \varepsilon_{3}(k), \varepsilon_{4}(k)$ and $\lambda_{i}(k)(i=1,2, \cdots, N)$ such that

$$
\begin{aligned}
& \Pi^{T}(k) P^{-1}(k+1) \Pi(k)-\operatorname{diag}\{1,0,0,0,0\} \\
& -\Gamma^{T}(k) \sum_{i=1}^{N} \lambda_{i}(k)\left(\bar{Q}\left(\Phi_{i}-\Phi_{\xi(k)}\right)\right) \Gamma(k) \\
& -\varepsilon_{1}(k) \operatorname{diag}\{-1, I, 0,0,0\}-\varepsilon_{2}(k) \operatorname{diag}\{-1,0, I, 0,0\} \\
& -\varepsilon_{3}(k) \operatorname{diag}\left\{-\tau_{2}, 0,0, I, 0\right\} \\
& -\varepsilon_{4}(k) \operatorname{diag}\left\{-2,0,0,0, \Omega^{-1}(k)\right\} \leq 0
\end{aligned}
$$

then the inequality $e^{T}(k+1) P^{-1}(k+1) e(k+1)-1 \leq 0$ can be guaranteed by the inequalities (21). The inequality (22) can be written in the following compact form:

$$
\begin{aligned}
& \Pi^{T}(k) P^{-1}(k+1) \Pi(k)-\mathcal{T} \\
& -\Gamma^{T}(k) \sum_{i=1}^{N} \lambda_{i}(k)\left(\bar{Q}\left(\Phi_{i}-\Phi_{\xi(k)}\right)\right) \Gamma(k) \leq 0 .
\end{aligned}
$$

According to the definition of $\Theta(k),(23)$ is rewritten as

$$
\Pi^{T}(k) P^{-1}(k+1) \Pi(k)-\Theta(k) \leq 0 .
$$

By using the Schur complement lemma, we have

$$
\left[\begin{array}{cc}
-\Theta(k) & \Pi^{T}(k) \\
* & -P(k+1)
\end{array}\right] \leq 0 .
$$

Thus, it can be obtained from (15) that the inequality $e^{T}(k+1) P^{-1}(k+1) e(k+1)-1 \leq 0$ is satisfied. Hence, by the induction, it can be concluded that the assertion is true for $k \in \mathbb{N}^{+}$, which means that the dynamics of the time-varying system (10) satisfies the $P(k)$-dependent constraint with the filter gain $K(k)$.

\section{2) The RR Protocol}

Theorem 2 Consider the system (4), the $R R$ protocol given by (1) and the filter (9). Let the sequence of constraint matrices $P(k)>0\left(k \in \mathbb{N}^{+}\right)$be given. If there exist real valued matrices $K(k)$, positive scalars $\varepsilon_{j}(k)$ $\left(k \in \mathbb{N}^{+}, j \in\{1,2,3,4\}\right)$ satisfying

$$
\left[\begin{array}{cc}
-\bar{\Theta}(k) & \Pi^{T}(k) \\
* & -P(k+1)
\end{array}\right] \leq 0
$$

where

$\bar{\Theta}(k)=\operatorname{diag}\left\{\bar{\varepsilon}(k), \varepsilon_{1}(k) I, \varepsilon_{2}(k) I, \varepsilon_{3}(k) I, \varepsilon_{4}(k) \Omega^{-1}(k)\right\}$ with other corresponding matrices defined in Theorem 1, then the dynamics of (10) satisfies the $P(k)$-dependent constraint with the estimator parameters $K(k)\left(k \in \mathbb{N}^{+}\right)$.

Proof: The proof is similar to that of Theorem 1 and is therefore omitted for the conciseness.

Remark 2 Sufficient conditions are provided in Theorem 1 and Theorem 2, respectively, under which the estimation error dynamics (10) satisfies the required $P(k)$ dependent constraint subject to the well-known WTOD protocol and $R R$ protocol. The main idea of the setmembership filter is to design the time-varying filter parameter $K(k)$ via the transmitted measurement output such that the estimation error is confined to the given ellipsoidal set at each time step. Obviously, the $P(k)$ dependent constraint (11) is equivalent to the quadratic error-bounded constraint $e(k) e^{T}(k) \leq P(k)$. 


\subsection{Design of Filter Gains Minimizing the Ellipsoids Subject to WTOD and RR Protocols}

In this subsection, two optimization problems are solved that would help the design of the optimal filter gains.

Corollary 1 Consider the dynamic system (4), the $W$ TOD protocol given by (3) and the time-varying filter (9). The ellipsoid constraint $P(k)$ on the estimation error is minimized (in the sense of the matrix trace) if there exist real-valued matrix $K(k)$, positive scalars $\lambda_{i}(k)\left(k \in \mathbb{N}^{+}\right.$, $i \in\{1,2, \cdots, N\})$ and $\varepsilon_{j}(k)\left(k \in \mathbb{N}^{+}, j \in\{1,2,3,4\}\right)$ solving the following optimization problem:

$$
\min _{P(k+1), K(k), \vec{\lambda}(k), \vec{\varepsilon}(k)} \operatorname{tr}\{P(k+1)\}
$$

subject to (15), where

$$
\vec{\lambda}(k)=\left\{\lambda_{i}(k)\right\}_{i \in\{1,2, \cdots, N\}}, \quad \vec{\varepsilon}(k)=\left\{\varepsilon_{i}(k)\right\}_{i \in\{1,2,3,4\}} .
$$

Corollary 2 For the dynamic system (4) subject to the $R R$ protocol (1), the ellipsoid constraint $P(k)$ on the estimation error is minimized (in the sense of the matrix trace) if there exist real-valued matrix $K(k)$ and positive scalars $\varepsilon_{j}(k)\left(k \in \mathbb{N}^{+}, j \in\{1,2,3,4\}\right)$ solving the following optimization problem:

$$
\min _{P(k+1), K(k), \vec{\varepsilon}(k)} \operatorname{tr}\{P(k+1)\}
$$

subject to (26), where $\vec{\varepsilon}(k):=\left\{\varepsilon_{i}(k)\right\}_{i \in\{1,2,3,4\}}$.

By means of Corollaries 1-2, we can summarize the Protocol-Based Set-Membership Filter Design (PBSMFD) algorithm as follows.

Algorithm PBSMFD:
Step 1. Initialization: Set $k=0$ and give the initial
constraint matrices $P(t)$ satisfying Assumption 2
for $t \in\left\{-\max \left\{\tau_{1}, \tau_{2}\right\},-\max \left\{\tau_{1}, \tau_{2}\right\}+1, \cdots, 0\right\}$.
Step 2. Calculate matrix factorizations $L(t)$ according to

$P(t)=L(t) L^{T}(t)$ for $k-\tau_{2} \leq t \leq k$ and $t=k-\tau_{1}$.
Step 3. Solve the corresponding optimization problem
according to the utilized scheduling protocol of the
network. If the network is scheduled under the
WTOD protocol, solve the optimization problem
(27) subject to (15). Otherwise, solve the
optimization problem $(28)$ subject to $(26)$. Then,
the filter parameter $K(k)$ and positive matrices

$P(k+1)$ are obtained according to the solution of
the optimization problem.
Step 4. Set $k=k+1$ and go to Step 2.

Remark 3 So far, we have discussed the set-membership filtering problems for a class of discrete time-varying systems with mixed time-delays subject to two kinds of communication protocols (the RR protocol and the $W T O D$ protocol). It can be observed from Algorithm $P B S M F D$ that, in the filter design procedure, all the important factors contributing to the system complexity are reflected in the main results which include 1) the time-varying system parameters; 2) the delay sizes; 3) the estimation accuracy (characterized by $P(k)$ ); 4 ) the noise information (characterized by $\mathscr{W}_{k}$ and $\mathscr{V}_{k}$ ); and 5) the information about the utilized communication protocol (determined by (1) or (3)).

\section{ILLUSTRATIVE EXAMPLES}

In this section, two numerical examples are presented to illustrate the effectiveness of the proposed setmembership filter design scheme for system (4) subject to the RR protocol and WTOD protocol, respectively.

Example 1: Consider a discrete-time delayed system (4) with the following system parameters:

$$
\begin{aligned}
A(k) & =\left[\begin{array}{cc}
0.75+0.35 \sin (0.4 k) & 0.02+0.01 \cos (0.2 k) \\
0.03 & 0.75+0.39 \sin (0.4 k)
\end{array}\right], \\
B(k) & =\left[\begin{array}{cc}
0.64 \\
0
\end{array}\right], E(k)=\left[\begin{array}{cc}
0.2 & 0 \\
0 & 0.2
\end{array}\right], D(k)=\left[\begin{array}{c}
0 \\
-0.4
\end{array}\right], \\
C(k) & =\left[\begin{array}{cc}
0.65 & -0.3 \\
-0.55 & 0.40
\end{array}\right], \quad F(k)=\left[\begin{array}{cc}
0.1 & 0 \\
0 & 0.1
\end{array}\right], \\
\tau_{1} & =1, \quad \tau_{2}=2, \quad \mu_{1}=\mu_{2}=0.2 .
\end{aligned}
$$

The sensors of this system are grouped into 2 sensor nodes. The weight matrices of the WTOD protocol is taken to be $Q_{1}=0.6$ and $Q_{2}=1.2$. The bounded noises are assumed to be $\omega(k)=0.9 \sin (k)$ and $\nu(k)=0.9 \cos (k)$, respectively. Obviously, the matrices $S(k)$ and $R(k)$ could be chosen as $S(k)=R(k)=0.81 I$. Also, select the initial state and constraint matrices as follows: $\psi(l)=\left[\begin{array}{llll}4 & 4 & 1 & 1\end{array}\right]^{T}, P(l)=\operatorname{diag}\{16,16,1,1\}$ $\left(l=-\max \left\{\tau_{1}, \tau_{2}\right\},-\max \left\{\tau_{1}, \tau_{2}\right\}+1, \cdots, 0\right)$.

Using the given algorithms and Matlab software (YALMIP 3.0), the set of solutions to the optimization problem (27) subject to (15) in Corollary 1 and the optimization problem (28) subject to (26) in Corollary 2 can be obtained, respectively. The simulation results are shown in Figs. 2-4. Figs. 2-3 depict the state responses and their estimations under the WTOD scheduling and RR scheduling. Fig. 4 plots the responses of the estimation error $e(k)$ with respect to the WTOD and RR protocols. All the simulation results confirm that the estimation performance is well achieved and the pronosed PBSMFD aløorithm is indeed effective.

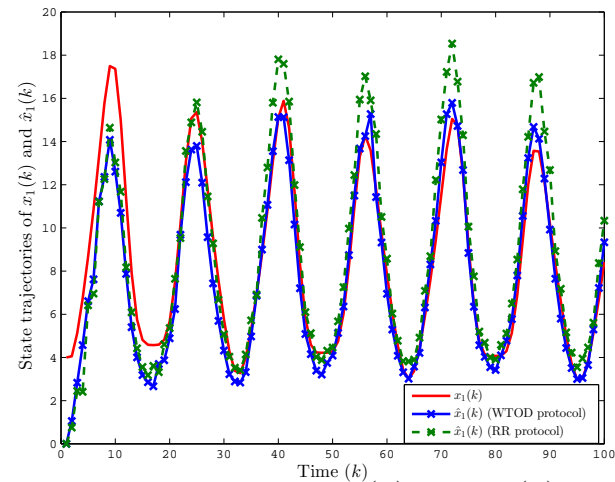

Fig. 2. The state trajectories of $x_{1}(k)$ and $\hat{x}_{1}(k)$ under the WTOD and RR protocols

Example 2: In this example, we consider a networkedbased test rig which consists of a plant (DC servo system) and a remote controller. The plant and the controller are connected via a communication network. The networked induced delay of such a communication network is 3 . Based on the results in [30], the DC servo system is identified to be a third-order system which can be described as follows: 


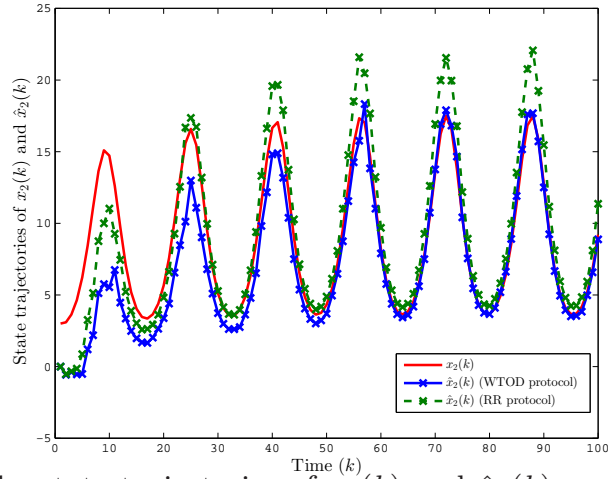

Fig. 3. The state trajectories of $x_{2}(k)$ and $\hat{x}_{2}(k)$ under the WTON and RR nrotonols

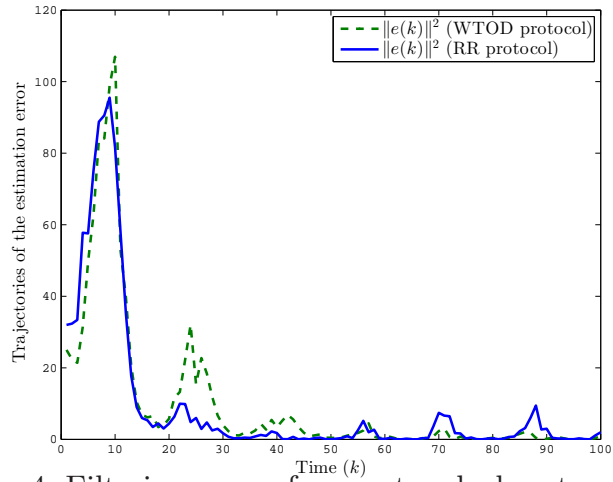

Fig. 4. Filtering errors for a networked system

$$
\left\{\begin{aligned}
x(k+1)= & {\left[\begin{array}{ccc}
1.12 & 0.213 & -0.333 \\
1 & 0 & 0 \\
0 & 1 & 0
\end{array}\right] x(k)+\left[\begin{array}{c}
0.8 \\
0 \\
0
\end{array}\right] \omega(k) } \\
& +\left[\begin{array}{ccc}
-0.2193 & 0.0219 & 0.0844 \\
0.2177 & -0.0032 & -0.0662 \\
0.1298 & -0.0087 & -0.0381
\end{array}\right] x(k-3) \\
y(k)= & {\left[\begin{array}{lll}
1 & 0 & 0 \\
0 & 1 & 0
\end{array}\right] x(k)+\left[\begin{array}{c}
0 \\
0.8
\end{array}\right] \nu(k) }
\end{aligned}\right.
$$

The sensors of this system are grouped into 2 sensor nodes and the weight matrices of the WTOD protocol is set to be $Q_{1}=0.8$ and $Q_{2}=1.2$. The process and measurement noise sequences are assumed to be $\omega(k)=0.5 \cos (0.2 k)$ and $\nu(k)=0.5 \sin (0.2 k)\left(k \in \mathbb{N}^{+}\right)$, respectively. Hence, the matrices $S(k)$ and $R(k)$ could be selected as $S(k)=R(k)=0.25 I$. The initial state and constraint matrices are chosen as follows: $\psi(l)=$ $\left[\begin{array}{lllll}2 & 2 & 2 & 1 & 1\end{array}\right]^{T}$ and $P(l)=\operatorname{diag}\{4,4,4,1,1\}(l=-1,0)$.

Based on the given algorithms and Matlab software (YALMIP 3.0), the set of solutions to the optimization problem (27) subject to (15) in Corollary 1 and the optimization problem (28) subject to (26) in Corollary 2 are obtained, respectively. The simulation results are shown in Figs. 5-8. Figs. 5-7 depict the state responses and their estimations under the WTOD scheduling and RR scheduling. Fig. 8 plots the responses of $e(k)$ with respect to the WTOD and RR protocols.

\section{CONCLUSION}

In this paper, the set-membership filtering problem has been investigated for a class of time-varying system with mixed-time-delays subject to the communication protocol scheduling. For the purpose of preventing the data from collisions, only one sensor node is permitted

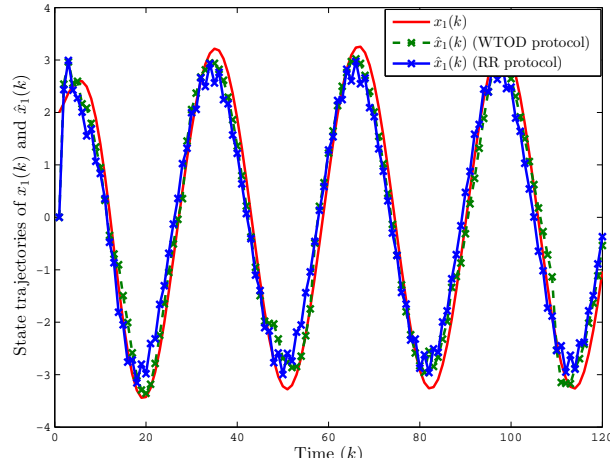

Fig. 5. The state trajectories of $x_{1}(k)$ and $\hat{x}_{1}(k)$ under the WTOD and RR protocols

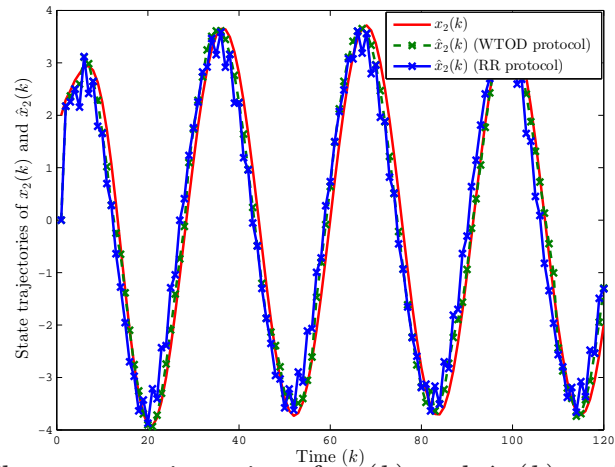

Fig. 6. The state trajectories of $x_{2}(k)$ and $\hat{x}_{2}(k)$ under the WTOD and RR protocols

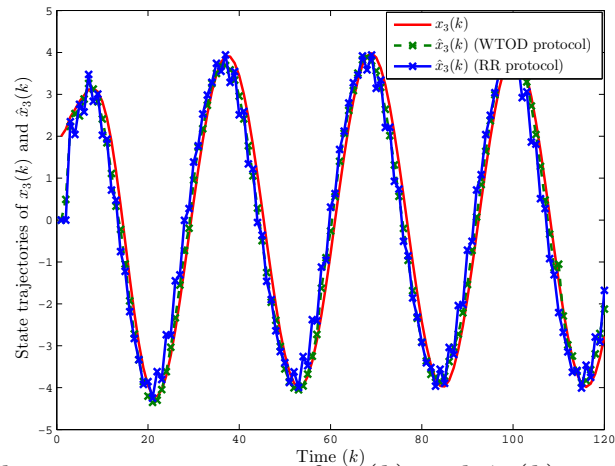

Fig. 7. The state trajectories of $x_{3}(k)$ and $\hat{x}_{3}(k)$ under the WTOD and RR protocols

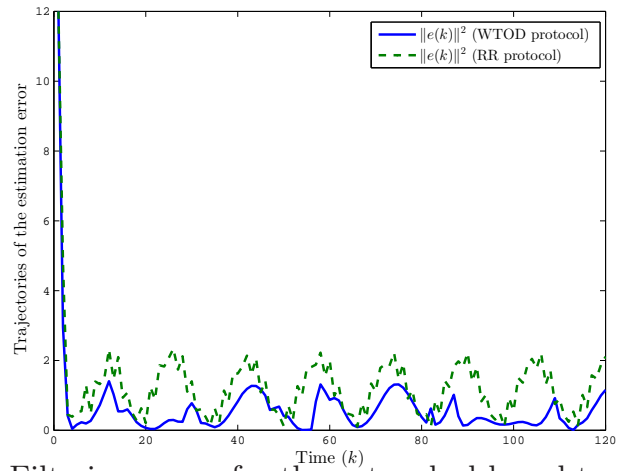

Fig. 8. Filtering errors for the networked-based test rig to get access to the shared network at each transmission instant. The transmission order of nodes is orches- 
trated by the communication protocol of the network. Two kinds of protocols (namely, the RR protocol and the WTOD protocol) have been studied respectively. A time-varying filter has been designed to obtain the estimate of the plant subject to the non-Gaussian noises and protocol scheduling. Sufficient conditions have been derived for the designed filter to satisfy the prescribed $P(k)$-dependent constraint in terms of recursive matrix inequalities. Within the established theoretical framework, two optimization problems have been proposed by optimizing the constraint ellipsoid of the estimation error subject to the aforementioned protocols. Finally, two illustrative examples have been given to highlight the effectiveness of the proposed filter design strategy.

\section{References}

[1] M. V. Basin, A. Loukianov and M. Hernandez-Gonzalez, "Joint state and parameter estimation for uncertain stochastic nonlinear polynomial systems", International Journal of Systems Science, vol. 44, no. 7, pp. 1200-1208, 2013.

[2] N. W. Bauer, M. C. F. Donkers, N. van de Wouw and W. P. M. H. Heemels, "Decentralized observer-based control via networked communication", Automatica, vol. 49, no. 7, pp. 2074-2086, Jul. 2013.

[3] S. Boyd, L. Ghaoui, E. Feron and V. Balakrishnan, Linear matrix inequalities in system and control theory, Philadelphia: SIAM Studies in Applied Mathematics, 1994.

[4] R. Caballero-Aguila, A. Hermoso-Carazo and J. LinaresPerez, "Optimal state estimation for networked systems with random parameter matrices, correlated noises and delayed measurements", International Journal of General Systems, vol. 44, no. 2, pp. 142-154, 2015.

[5] D. Ding, Z. Wang, B. Shen, and G. Wei, "Eventtriggered consensus control for discrete-time stochastic multiagent systems: the input-to-state stability in probability", Automatica, vol. 62, pp. 284-291, 2015.

[6] L. El Ghaoui and G. Calafiore, "Robust filtering for discrete-time systems with bounded noise and parametric uncertainty", IEEE Transactions on Automatic Control, vol. 46, no. 7, pp. 1302-1313, Jul. 2001.

[7] M. C. F. Donkers, W. P. M. H. Heemels, N. van de Wouw and L. Hetel, "Stability analysis of networked control systems using a switched linear systems approach", IEEE Transactions on Automatic Control, vol. 56, no. 9, pp. 21012115, Sep. 2011.

[8] L. Guo and H. Wang, "Minimum entropy filtering for multivariate stochastic systems with non-Gaussian noises", IEEE Transactions on Automatic Control, vol. 51, no. 4, pp. 695-700, Apr. 2006.

[9] L. Guo and H. Wang, "Frequency-domain set-membership filtering and its applications", IEEE Transactions on Signal Processing, vol. 55, no. 4, pp. 1326-1338, Apr. 2007.

[10] N. Hou, H. Dong, Z. Wang, W. Ren and F. E. Alsaadi, "Non-fragile state estimation for discrete Markovian jumping neural networks", Neurocomputing, vol. 179, pp. 238-245, 2016.

[11] J. Hu, Z. Wang, H. Gao and L. K. Stergioulas, "Robust sliding mode control for discrete stochastic systems with mixed time delays, randomly occurring uncertainties, and randomly occurring nonlinearities", IEEE Transactions on Industrial Electronics, vol. 59, no. 7, pp. 3008-3015, 2012.

[12] J. Lam, B. Zhang, Y. Chen and S. Xu, "Reachable set estimation for discrete-time linear systems with time delays", International Journal of Robust and Nonlinear Control, vol. 25, no. 2, pp. 269-281, 2015.
[13] G. Liu, Y. Xia, J. Chen and W. Hu, "Networked predictive control of systems with random network delays in both forward and feedback channels", IEEE Transactions on Industrial Electronics, vol. 54, no. 3, pp. 1282-1297, Jun. 2007.

[14] Y. Long and G.-H. Yang, "Fault detection and isolation for networked control systems with finite frequency specifications", International Journal of Robust and Nonlinear Control, vol. 24, no. 3, pp. 495-514, Feb. 2014.

[15] H. Song, L. Yu and W. Zhang, "Stabilisation of networked control systms with communciation constraints and multiple distributed transmission delays", IET Control Theory and Applications, vol. 3, no. 10, pp. 1307-1316, Oct. 2009.

[16] M. Tabbara and D. Nešić, "Input-output stability of networked control systems with stochastic protocols and channels", IEEE Transactions on Automatic Control, vol. 53, no. 5, pp. 1160-1175, Jun. 2008.

[17] V. Ugrinovskii and E. Fridman, "A Round-Robin type protocol for distributed estimation with $\mathcal{H}_{\infty}$ consensus", Systems \& Control Letters, vol. 69, pp. 103-110, Jul. 2014.

[18] G. C. Walsh, H. Ye and L. G. Bushnell, "Stability analysis of networked control systems", IEEE Transactions on Control Systems Technology, vol. 10, no. 3, pp. 438-446, May. 2002.

[19] G. Wei, S. Liu, Y. Song and Y. Liu, "Probability-guaranteed set-membership filtering for systems with incomplete measurements", Automatica, vol. 60, pp. 12-16, Jul. 2015.

[20] J. Xiong and J. Lam, "Stabilization of linear systems over networks with bounded packet loss", Automatica, vol. 43, no. 1, pp. 80-87, Jan. 2007.

[21] J. Xiong and J. Lam, "Stabilization of networked control systems with a logic ZOH", IEEE Transactions on Automatic Control, vol. 54, no. 2, Feb. 2009.

[22] Y. Xu, H. Su, Y. Pan, Z. Wu and W. Xu, "Stability analysis of network control systems with round-robin scheduling and packet dropouts", Journal of The Franklin Institute, vol. 350, no. 8, pp. 2013-2027, Oct. 2013.

[23] F. Yang, H. Dong, Z. Wang, W. Ren and F. E. Alsaadi, "A new approach to non-fragile state estimation for continuous neural networks with time-delays", Neurocomputing, vol. 197, pp. 205-211, 2016.

[24] F. Yang and Y. Li, "Set-membership filtering for discretetime systems with nonlinear equality constraints", IEEE Transactions on Automatic Control, vol. 54, no. 10, pp. 24802486, Oct. 2009

[25] F. Yang and Y. Li, "Set-membership filtering for systems with sensor saturation", Automatica, vol. 45, no. 8, pp. 18961902, Aug. 2009.

[26] Y. Yu, H. Dong, Z. Wang, W. Ren and F. E. Alsaadi, "Design of non-fragile state estimators for discrete timedelayed neural networks with parameter uncertainties", Neurocomputing, vol. 182, pp. 18-24, 2016.

[27] L. Zhang, Y. Shi, T. Chen and B. Huang, "A new method for stabilization of networked control systems with random delays", IEEE Transactions on Automatic Control, vol. 50, no. 8, pp. 1177-1181, Aug. 2005.

[28] S. Zhang, Z. Wang, D. Ding, H. Dong, F. E. Alsaadi and T. Hayat, "Nonfragile $H_{\infty}$ fuzzy filtering with randomly occurring gain variations and channel fadings", IEEE Transactions on Fuzzy Systems, vol. 24, no. 3, pp. 505-518, Jun. 2016.

[29] W. Zhang, L. Yu and G. Feng, "Optimal linear estimation for networked systems with communication constraints", Automatica, vol. 47, no. 9, pp. 1992-2000, Sep. 2011.

[30] Y. Zhao, G. Liu and D. Rees, "Design of a packet-based control framework for networked control systems", IEEE Transactions on Control Technology, vol. 17, no. 4, pp. 859865, Jul. 2009. 